\title{
Spherical accretion in nearby weakly active galaxies
}

\author{
M. Moscibrodzka
}

N. Copernicus Astronomical Center, Bartycka 18, 00-716, Warsaw, Poland

e-mail: mmosc@camk.edu.pl

Received 6 September 2005 / Accepted 15 December 2005

\section{ABSTRACT}

We consider the sample of weakly active galaxies situated in the "Local Universe" with inferred accretion efficiencies from $10^{-2}$ to $10^{-7}$. We apply a model of spherically symmetrical Bondi accretion for given parameters $\left(M_{\mathrm{BH}}, T_{\infty}, \rho_{\infty},\right)$ taken from observation. We calculate spectra emitted by the gas accreting onto its central objects using a Monte Carlo method including synchrotron and bremsstrahlung photons as seed photons. We then compare our results with observed nuclear X-ray luminosities $L_{\mathrm{X} \text {, nuc }}(0.3-10 \mathrm{keV})$ for the sample. A model is also tested for different external medium parameters $\left(\rho_{\infty}\right.$ and $T_{\infty}$ ) and different free parameters of the model. Our model is able to explain observed nuclear luminosities $L_{\mathrm{X}}$ under an assumption that half of the compression energy is transferred directly to the electrons.

Key words. galaxies: nuclei - galaxies: active - galaxies: cooling flows

\section{Introduction}

It is believed that most of the galactic nuclei host super-massive black holes in their centers. The masses of these central objects estimated by various methods are of the order on $10^{6}-10^{9} M_{\odot}$. The radiation emerging from the vicinity of these objects is produced by an accretion process. We believe that quasars and Seyfert galaxies are powered by the flows with high angular momentum and are characterized by high accretion efficiency. Corresponding models consisting of a thin accretion disk surrounded by some kind of corona are able to explain all spectral features like big blue bump, iron line, and short time-scale variability. The accretion luminosity in these sources is on order of a few percent of the Eddington limit or more.

Nevertheless a number (40\% of nearby galaxies, Ho 2003) of galactic nuclei exist that are very faint objects, although they contain black holes of masses around as those in AGN. For example the Galactic center hosts a super-massive object of mass $\sim 10^{6} M_{\odot}$ (Genzel et al. 2003). The X-ray luminosity of this object is about $10^{-9}$ times smaller than its Eddington limit. The density and the temperature near the capture radius were estimated from Chandra data (Baganoff et al. 2003), which allowed computation the expected mass accretion rate, and calculation of the dynamics of the accreting gas using, for example, the simplest Bondi model (1952) of the steady spherically symmetric flow. The efficiency of this accretion flow, as estimated by comparing the expected accretion rates and observed luminosities, is about $\eta=10^{-5}$, which is very small compared to the typical active quasar value where efficiency is $\eta=0.1$.

The Galactic center is an example of the extremely faint source, sometimes it is even called inactive (Nayakshin 2004).
In the paper by Pellegrini (2005, hereafter P2005), more examples are collected of faint galactic nuclei sources (LLAGN, low luminosity active galactic nuclei) located in the "Local Universe". The masses of black holes in centers of these objects obtained by various methods (for details see P2005) lie in the same range as typical $M_{\mathrm{BH}} \mathrm{AGN}$. Their luminosities are approximately $10^{-2}-10^{-7}$ of the Eddington luminosity. The efficiency of the accretion (assuming Bondi mass accretion rate) is thus also low. The LLAGN have no big blue bump (Quataert et al. 1999), only weak iron lines, and no short time-scale variability (Ptak et al. 1998), which is evidence of the nonexistence of the accretion disk inside the flow (Nayakshin 2004, and references therein). To build a consistent picture, we would like those objects to be quiescent phases of long-term evolution of quasars (Nayakshin 2004). To explain such low activity we usually assume one of the RIAF (radiatively inefficient accretion flow) solutions. Examples of RIAFs include Bondi flow with no angular momentum, low angular momentum flows (Proga 2003), and ADAF with high angular momentum (Narayan 2002). Other possibilities are spherical flows with magnetic fields, convection dominated flows (Narayan 2002, and references therein), or jet-disk accretion flows (Yuan et al. 2003a).

In this paper, to explain low luminosities of the sample of LLAGN, we apply the spherically symmetrical accretion in the Newtonian regime (Bondi 1952). In this model, the accretion rate is determined by external medium conditions and remain constant in the whole radius range, thus we do not include outflow in any form. Bondi flow can be also treated as a rough approximation of very low angular momentum flows in a steady phase. Plasma accreting onto a central object in our model is a source of synchrotron and bremsstrahlung photons. 
We assume that plasma has a two-temperature structure. Because in our model cooling of the flow occurs by electrons, ions are much hotter than electrons near the horizon. In calculations of emerging radiation spectrum we include the density and the temperatures of ions and electrons as a function of radius. Radiative transfer of synchrotron and bremsstrahlung radiation through the plasma is calculated using Monte Carlo technique (Gorecki \& Wilczewski 1984; Pozdnyakov et al. 1983). Because of the method of calculating the spectra, we ignore the radiation pressure influence on the flow dynamics.

In Sect. 2 we describe the model and the technique of calculating the Comptonization in detail. We present the result in Sect. 3. Discussion is given in Sect. 4.

\section{Model}

\subsection{Dynamics of the flow}

The dynamics of the flow is given by Bondi solution (Bondi 1952). We assume the black hole mass $M_{\mathrm{BH}}$, density of the matter at the infinity $\rho_{\infty}$, and temperature at infinity $T_{\infty}$ to calculate the density of matter and velocity of infall profile. No additional sources of the gas or outflow are included. The polytropic index $\gamma$ is assumed to be equal when close to $\frac{5}{3}$. This is a reasonable assumption since matter in the close vicinity of a black hole is fully ionized. The Bondi radius is then very close to a black hole, on the order of a few Schwarzschild radii. The flow is then subsonic in almost the whole range.

\subsection{Temperature of the electrons and ions}

To calculate the ion and electron temperature we use the iterative procedure given by Esin et al. (1997). At first we assume a reasonable electron temperature $T_{\text {e }}$ profile and calculate the energy balance at each point of the flow. The ion temperature is determined simultaneously with the corrections to the electron temperature $T_{\mathrm{e}}$ from the relation

$$
P_{\text {gas }}=\beta \rho c_{\mathrm{s}}^{2}=n_{\mathrm{i}} k_{\mathrm{b}} T_{\mathrm{i}}+n_{\mathrm{e}} k_{\mathrm{b}} T_{\mathrm{e}}
$$

where $\beta$ is the gas to total pressure ratio, $n_{\mathrm{e}}$ and $n_{\mathrm{i}}$ are the total numbers per $\mathrm{cm}^{3}$ of electrons and ions, respectively. The condition of the thermal equilibrium for electrons is given by:

$Q_{\mathrm{adv}}=Q^{\mathrm{ie}}+\delta Q_{\mathrm{grav}}-Q^{\mathrm{rad}}$.

We have the advection term on the left hand side. On the right there are the heating rate of the electrons by a Coulomb coupling, accretion energy (compression heating) multiplied by a small $\delta$ parameter, and a cooling rate. The heating rate due to the Coulomb coupling between ions and electrons is given by (e.g. Rozanska et al. 2000):

$q^{\text {ie }}=1.5 k_{\mathrm{b}} m_{\mathrm{H}} \ln \Lambda \rho^{2} 2.44 \times 10^{21} T_{\mathrm{e}}^{-1.5}\left(T_{\mathrm{p}}-T_{\mathrm{e}}\right)$.

Cooling rate $Q^{\text {rad }}$ consists of four terms: the synchrotron cooling and comptonization of synchrotron photons cooling, bremsstrahlung cooling, and comptonization of bremsstrahlung:

$Q^{\mathrm{rad}}=q_{\mathrm{S}}^{-}+q_{\mathrm{C}, \mathrm{S}}^{-}+q_{\mathrm{brem}}^{-}+q_{\mathrm{C}, \mathrm{brem}}^{-}$.
The derivation of $q_{\mathrm{C}, \mathrm{S}}^{-}$and $q_{\mathrm{C} \text {,brem }}^{-}$is described in Esin et al. (1996) and the cooling by the synchrotron photons is given by:

$q_{\mathrm{S}}^{-}=\int_{0}^{\infty} \epsilon_{\mathrm{S}}(v) \mathrm{e}^{-\tau_{v}} \mathrm{~d} v$

The exponential term is due to a strong self-absorption of the synchrotron photons below a given frequency. The optical depth is a function of frequency, so we can calculate it by estimating the escape probability of the photon of given energy from a given location. To find this probability we follow $N$ trajectories of photons (rays) with randomly chosen directions. We follow the rays and calculate the optical depth, which is given by:

$\tau_{v}=\int_{0}^{r_{\text {boundary }}} \alpha_{\nu}^{\mathrm{S}} \mathrm{d} l=\int_{0}^{r_{\text {boundary }}} \frac{\epsilon_{S}(v)}{4 \pi B_{T_{\mathrm{e}}}(v)} \mathrm{d} l$.

Here $\alpha_{v}^{\mathrm{S}}$ is the absorption coefficient, and the second part of the Eq. (6) is a Kirchoff's law. The mean optical depth is

$\hat{\tau}_{v}=\frac{1}{N} \sum_{i=1}^{N} \mathrm{e}^{-\tau_{v}^{\mathrm{i}}}$.

This procedure of deriving $q^{-}$is described in more detail in Kurpiewski et al. (2001).

In the thermal balance equation we assume that part of the energy must be advected without emission. The term $Q_{\mathrm{adv}}$ is calculated as described e.g. in Narayan et al. (1998). We also assume that part of the compression energy taken by ions is taken by the electrons. This is given by $\delta Q_{\text {grav }}$ where $\delta$ in a parameter of this model. This energy is proportional to the temperature of ions, the density, and the velocity of the falling matter $\left(v=v_{r}\right)$. It is given by

$Q_{\text {grav }}=\frac{3}{2} \frac{k_{\mathrm{b}} T_{\mathrm{i}} \rho}{\mu m_{\mathrm{H}} r} v$

Corrections to the electron temperature are calculated from energy balance equation iteratively, until they are less that $1 \%$ of the $T_{\mathrm{e}}$.

\subsection{Emissivity of plasma}

We take bremsstrahlung photons and synchrotron photons into account. The emissivity of synchrotron is given by Pacholczyk (1970) formula

$\epsilon_{\mathrm{S}}(v)=4.43 \times 10^{-30} \frac{4 \pi v n_{\mathrm{e}}}{K_{2}(1 / \theta)} I\left(\frac{x_{\mathrm{M}}}{\sin \Phi}\right)\left[\mathrm{ergs} / \mathrm{s} / \mathrm{cm}^{3} / \mathrm{Hz}\right]$

where

$x_{\mathrm{M}}=\frac{2 v}{3 v_{0} \theta_{2}} \quad v_{0}=\frac{e B}{2 \pi m_{\mathrm{e}} c}$.

Here $\theta$ is dimensionless electron temperature, $B$ is the magnetic field, $v_{0}$ is the cyclotron frequency, $\Phi$ is the angle between the velocity vector and the direction of local magnetic field. The $B$ field is calculated assuming beta parameter defined as 
$\beta=P_{\text {gas }} / P_{\text {tot }}$. For the isotropic velocity function we can average $I\left(\frac{x_{M}}{\Phi}\right)$ over $\Phi$ angle. The averaged function was given by Mahadevan et al. (1996):

$I^{\prime}\left(x_{\mathrm{M}}\right)=\frac{4.0505}{x_{\mathrm{M}}^{1 / 6}}\left(1+\frac{0.40}{x_{\mathrm{M}}^{1 / 4}}+\frac{0.5316}{x_{\mathrm{M}}^{1 / 2}}\right) \exp \left(-1.8899 x_{\mathrm{M}}^{1 / 3}\right)$.

Because the approximation above is good for ultra-relativistic electrons, additionally for temperatures $\left(T_{\mathrm{e}}<10^{10} \mathrm{~K}\right)$ we also use the modified Eq. (11):

$$
\begin{aligned}
& I^{\prime}\left(x_{\mathrm{M}}\right)= \\
& \quad \frac{4.0505 \alpha^{\prime}}{x_{\mathrm{M}}^{1 / 6}}\left(1+\frac{0.40 \beta^{\prime}}{x_{\mathrm{M}}^{1 / 4}}+\frac{0.5316 \gamma^{\prime}}{x_{\mathrm{M}}^{1 / 2}}\right) \exp \left(-1.8899 x_{\mathrm{M}}^{1 / 3}\right) .
\end{aligned}
$$

The coefficients are derived from comparing the synchrotron emissivity with the cyclo-synchrotron emissivity. We take these coefficients from Mahadevan et al. (1996) and interpolate them. For ultra-relativistic temperatures, the $\alpha^{\prime}, \beta^{\prime}$ and $\gamma^{\prime}$ coefficients approach 1 . The self-absorption of the synchrotron photons is described in the previous subsection, and the bremsstrahlung emissivity is given by:

$\epsilon_{v}^{f f}=q_{\mathrm{br}}^{-} \mathrm{e}^{-h v / k_{\mathrm{b}} T} \bar{g}_{f f}\left[\mathrm{erg} / \mathrm{s} / \mathrm{cm}^{3} / \mathrm{Hz}\right]$

where $q_{\mathrm{br}}^{-}=q_{\mathrm{ie}}+q_{\mathrm{ee}}^{-} \cdot q_{\mathrm{ie}}$ is for ion-electron interaction and $q_{\mathrm{ee}}$ is for electron-electron interaction. Electrons are radiating in these two processes. The formulas for these emissivities are standard ones taken from e.g. Narayan et al. (1995). We also include the absorption of bremsstrahlung emission, which is calculated in the same manner as for synchrotron radiation.

\subsection{Photon generation}

The Monte Carlo method given by Gorecki \& Wilczewski (1984) is based on following the photons (or rays) trajectories. At first we generate some initial values of the photon energy, place of birth, and the direction in which the photon is emitted.

- Photon energy. The photon energy is randomly generated from the photon distribution in a given location. The photon distribution is given by the energy distribution divided by $h v$ and additionally multiplied by the $\exp \left(-\tau_{v}\right)$ that indicates the photon self-absorption. The photon distribution is given by

$f_{i, j, l}=\frac{n_{v}\left(r_{i}, \theta_{j}, \phi_{l}\right)}{\int_{0}^{\infty} n_{v}\left(r_{i}, \theta_{j}, \phi_{l}\right) d v}$,

where $i, j, l$ numbers indicate the cell numbers (see Sect. 2.7), which correspond respectively to radius $r, \theta$ and $\phi$ angle.

$n_{v}\left(r_{i}, \theta_{j}, \phi_{l}\right)=\frac{\epsilon_{v}\left(r_{i}, \theta_{j}, \phi_{l}\right) \mathrm{e}^{-\tau_{v}}}{h v}$.

The initial photon energy $h v_{0}$ is determined by the inversion of the cumulative distribution function.

- Initial position vector. The initial position of the emission of the photon is given by the distribution that is the integrated photon distribution from zero to infinity in a frequency

$$
\begin{aligned}
& f_{i, j, k}=\frac{\dot{N}_{i, j, l}}{\sum_{i, j, l} \dot{N}_{i, j, l}} \\
& \dot{N}_{i, j, l}=\Delta V_{i, j, l} \int_{0}^{\infty} \frac{\epsilon(v) \mathrm{e}^{-\hat{\tau}_{v}}}{h v} \mathrm{~d} v,
\end{aligned}
$$

where $\Delta V_{i, j, l}$ is the cell volume. The angles $\cos \theta$ and $\phi$ are generated from uniform distributions in the ranges $\cos \theta \in$ $(-1,1)$ and $\phi \in(0,2 \pi)$.

- Direction of emission. The direction of emission is randomly chosen from uniform distributions. The direction is given by a vector $\boldsymbol{\Omega}=(\sin \Theta \cos \phi, \sin \Theta \sin \phi, \cos \Theta)$, where: $\cos \Theta \in[-1,1]$ and $\phi \in[0 ; 2 \pi]$.

- Weight of the ray. The initial weight is $w_{0}=1$, and we follow the trajectory of a ray until $w=w_{\min }$. In our calculations we adopt $w_{\min }=10^{-7}$ (Kurpiewski et al. 2001).

\subsection{Photon propagation}

To count the spectrum we follow the method of Gorecki $\&$ Wilczewski. Each ray initially has a weight of $w_{0}=1$, energy $h v_{0}$, direction $\Omega_{0}$, and it is emitted from $\mathbf{r}_{0}$ location. We construct vector $T_{i}=\left(\boldsymbol{r}_{i}, \boldsymbol{\Omega}_{i}, h v_{i}, w_{i}\right)$, where $T_{0}=$ $\left(\boldsymbol{r}_{0}, \boldsymbol{\Omega}_{0}, h v_{0}, w_{0}\right)$. Knowing the zero order values we can perform the next step. To determine the emerging spectrum we calculate the probability that the photon leaves the cloud, which is given by:

$P\left(l_{i}\right)=\exp \left(-\int_{0}^{l_{i}} N_{\mathrm{e}}\langle\sigma\rangle \mathrm{d} l\right)$,

where $l_{i}$ is the distance to the boundary of the cloud from a given location (a place of last scattering) in a chosen direction. The value $N_{\mathrm{e}}$ is the electron density and $\langle\sigma\rangle$ is the cross section for scattering given by:

$\langle\sigma\rangle=\frac{1}{N_{\mathrm{e}}} \int N(\boldsymbol{v})(1-\boldsymbol{v} \cdot \mathbf{\Omega} / c) \sigma(x) \mathrm{d}^{3} v$.

The cross section is also averaged by the term that takes the probability of the photon-electron interaction into account. To obtain emerging spectra we count $P_{i} w_{i}$ in energy bands. The next value of $w_{i+1}=w_{i}\left(P_{i}-1\right)$. To obtain the position of the next scattering we model the mean free path distribution. It is given by

$\rho\left(\lambda_{i}\right)=\frac{\frac{\mathrm{d} \tau\left(\lambda_{i}\right)}{\mathrm{d} \lambda_{i}} \mathrm{e}^{-\tau\left(\lambda_{i}\right)}}{\int_{0}^{\lambda_{i}} \frac{\mathrm{d} \tau\left(\lambda_{i}\right)}{\mathrm{d} \lambda_{i}} \mathrm{e}^{-\tau\left(\lambda_{i}\right)} \mathrm{d} \lambda}$

where

$\tau(\lambda)=\int_{0}^{\lambda} N e\langle\sigma\rangle \mathrm{d} x$

The next collision vector is given by:

$\boldsymbol{r}_{i+1}=\boldsymbol{r}_{i}+\lambda_{i} \mathbf{\Omega}_{i}$,

where the $\boldsymbol{\Omega}_{i+1}$ vector is generated from uniform distributions of $\cos \Theta$ and $\phi$. The energy of a new photon after collision is given by standard formulas; for details on how to model the distributions, see Gorecki \& Wilczewski (1984). 


\subsection{Seed photons}

To include both synchrotron and bremsstrahlung emission, we count both contributions to spectra separately. After that we add spectra to each other with proper weights. The total number of emitted synchrotron photons is given by

$N_{\mathrm{S}}=\int \frac{\epsilon_{\mathrm{S}}(v)}{h v} \mathrm{e}^{-\tau_{\text {synch }}} \mathrm{d} v$

and the total number of bremsstrahlung photons produced in the flow is

$N_{\mathrm{B}}=\int \frac{\epsilon_{\mathrm{B}}(v)}{h v} \mathrm{e}^{-\tau_{\text {brem }}} \mathrm{d} \nu$.

We calculate this integral and obtain the weight of the bremsstrahlung photons, which is given by:

$\eta_{\mathrm{B}-\mathrm{S}}=\frac{N_{\mathrm{B}}}{N_{\mathrm{S}}}$.

To obtain the whole spectrum we add the synchrotron spectrum to the bremsstrahlung spectrum multiplied by factor $\eta_{\mathrm{B}-\mathrm{S}}$.

\subsection{Numerical calculations and parameters of the model}

In our calculations we divide the flow into cells. The geometry of the flow is represented in spherical coordinates. We divide the flow in radius in logarithmic scale and angles $\cos \Theta$ and $\phi$. The flow is divided into $10^{6}(100 \times 100 \times 100)$ cells. We assume that the value in the middle of the cell of the quantities is the mean value in the whole cell. The code was tested with homogeneous plasma, with central source of black body photons, and also for ADAF solutions. It gave similar results to in other papers by Gorecki \& Wilczewski (1984) and Narayan et al. (1995). The difference between Narayan et al. (1995) and our approach is that we include the self-absorption of bremsstrahlung photons. Also the results are slightly different because in ADAF the authors use different geometry. The code also can be used for any dynamical solution. The parameters of the model are: the black hole mass $M_{\mathrm{BH}}$, initial values at infinity $\rho_{\infty}, T_{\infty}$, and $\gamma$. The first three parameters are taken from observations of a given source. The parameter $\gamma$ is been assumed to be close to $5 / 3$ which is good for the fully ionized plasma (in calculations $\gamma=1.666$, because of numerical difficulties). Then $\beta$ and $\delta$ are free parameters that influence the electron temperature. The outer boundary of the spherical cloud is chosen as the be Bondi radius. The boundary of the flow is also a parameter of the model. The inner radius of the Comptonizing cloud is always fixed at the 3 Schwarzschild radii.

\section{Results}

\subsection{Model parameter tests}

In general the radiation spectra emitted from the vicinity of the central objects in our model consist of two components. First, one is created by synchrotron photons, and the second by bremsstrahlung photons. Most of the synchrotron photons

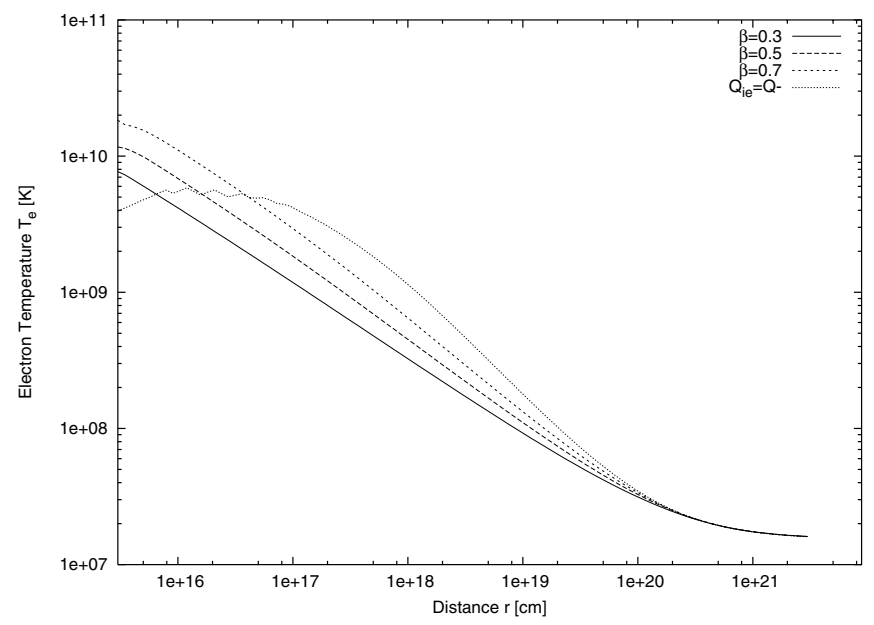

Fig. 1. Electron temperature profiles for different model parameters $\beta$, for fixed $\delta=0.001$.

emerge from the spherical cloud of plasma without scattering and thus create a bump in radio band. The synchrotron bump in some cases can be shifted to IR band depending on the parameters of the model $\left(M_{\mathrm{BH}}, \rho_{\infty}, T_{\infty}, \beta, \delta\right)$. The second, weaker bump in the radio-IR-opt-UV range is created by synchrotron photons that run out the spherical cloud of plasma after first scattering. The frequency range in which the bump, created by the single scattered photons, is exactly located also depends on the parameters of the model. Because of the low optical depth (in all cases $\tau<1$ ) of the accreting gas, the bump created by photons after first scattering is lower than the main synchrotron peak. The probability that photons undergo $k$ th scattering before escaping the cloud is proportional to $\tau^{k}$. The $\mathrm{X}-\gamma$ ray bump is mostly created by bremsstrahlung photons. It can be affected by the synchrotron photons scattered more than once, if the plasma optical thickness is high enough.

\subsubsection{Results for different $\beta$ parameters}

To test the model for different parameters in all its details, we performed calculations for the case of M 87. The electron temperature profiles for different $\beta$ values $(\beta=0.3,0.5,0.7)$ for this source are presented in Fig. 1. We assume that electrons receive only a small fraction of the compression energy, and we set $\delta=0.001$. Because of the ratio $m_{\mathrm{e}} / m_{\mathrm{p}}$, in our basic model we assume that compression energy is mostly taken by ions, because they are more massive than electrons, and also perform calculations for other values of $\delta$. For comparison in Fig. 1, we also plot the electron temperature profile obtained by iterating Eq. (3) without advection $Q_{\mathrm{adv}}$ and compression heating $\delta Q_{\text {grav }}$ terms, marked as $Q_{\text {ie }}=Q^{-}$.

In Fig. 2 we present how four terms of heating $\left(Q_{\text {ie }}, \delta Q_{\text {grav }}\right)$ and cooling $\left(Q^{-}, Q_{\mathrm{adv}}\right)$ balance each other for $\beta=0.5$ and $\delta=$ 0.001. Far away from a horizon the dominating terms in Eq. (3) are electron-ion coupling and radiation cooling. The electron temperature is the same there as when we neglect advection and compression heating terms. Close to the black hole the electron temperature is determined by the advection term, which dominates in this region. The advection term itself consists of 




Fig. 2. Heating and cooling terms as a function of radius for parameter $\delta=0.001$ and $\beta=0.5$.

two parts that balance each other out. The other terms are negligible near the black hole. Electrons reach higher temperatures close to the horizon than in the case where we balance only $Q_{\text {ie }}$ and $Q^{-}$. On the other hand, in the case where the energy balance equation is $Q_{\mathrm{ie}}=Q^{-}$, electrons are hotter farther from the black hole. The problem of iterating the electron temperature in case when the advection term dominate, becomes local. The non-locality caused by the radiative cooling term $Q^{-}$is now negligible.

An increase in the $\beta$ parameter causes the decrease in the magnetic field and an increase in the electron temperature. The spectra calculated for the temperature profiles presented in Fig. 1 are plotted in Fig. 5. The calculations were performed for the boundary radius of the cloud equal to Bondi radius. The differences among these cases mainly concerns the synchrotron part of the spectrum. The synchrotron emissivity increases for higher $\beta$ values, because most of the synchrotron photons are created very close to the black hole where the temperatures differ the most. The $L_{\mathrm{X}}$ luminosities resulting from integrating the outgoing radiation spectrum in range $0.3-10 \mathrm{keV}$ do not depend strongly on the $\beta$ parameter. The X-ray band of the spectrum is dominated by the bremsstrahlung photons created farther form a black hole. Calculated $\log _{10}\left(L_{\mathrm{X}}\right)$ for $\beta=0.3,0.5$, 0.7 are, respectively, $37.0,37.15,37.42$, where $L_{X}$ is meassured in ergs/s. In the case of $\beta=0.7$, the X-ray band is affected a little by synchrotron photons scattered in the medium more than once. For the $Q_{\mathrm{ie}}=Q^{-}$case, synchrotron emissivity is much lower, because the temperature near the horizon is smaller. The value of $\log _{10}\left(L_{\mathrm{X}}\right)=37.44$ in this case is larger because at larger distances the electron temperature is higher.

\subsubsection{Results for different $\delta$ parameters}

Since the representative value of $\delta$ is disputable (e.g. Yuan et al. 1995; Bisnovatyi-Kogan \& Lovelace 1997; Narayan \& Yi 2003), in Fig. 3 we show the effect of changing the $\delta$ parameter in Eq. (3). We made calculations for four values of $\delta$, namely $0.001,0.01,0.1$, and 0.5 , as $\beta$ is set as 0.5 . For larger $\delta$ more compression energy is put into electrons. The largest difference

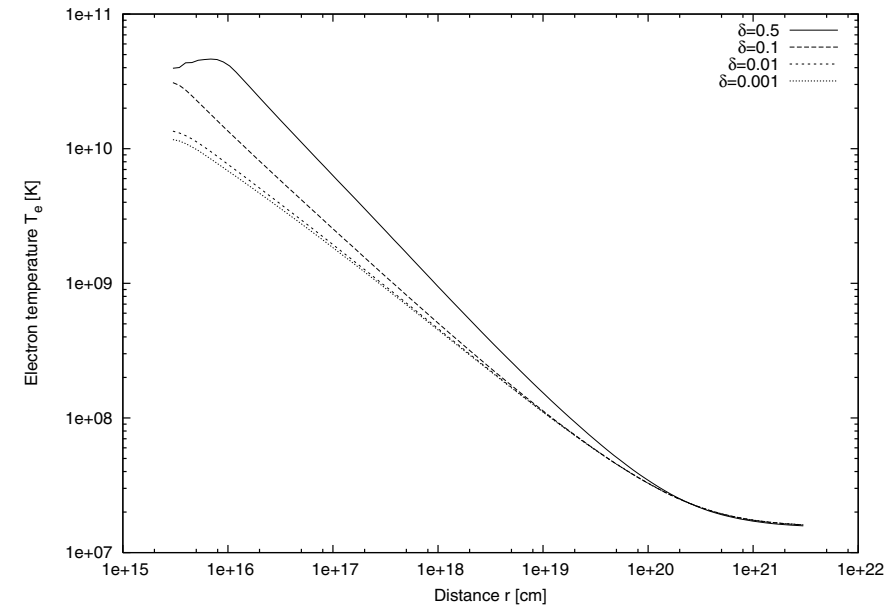

Fig. 3. Electron temperature profiles for different model parameters $\delta$ with fixed $\beta=0.5$.

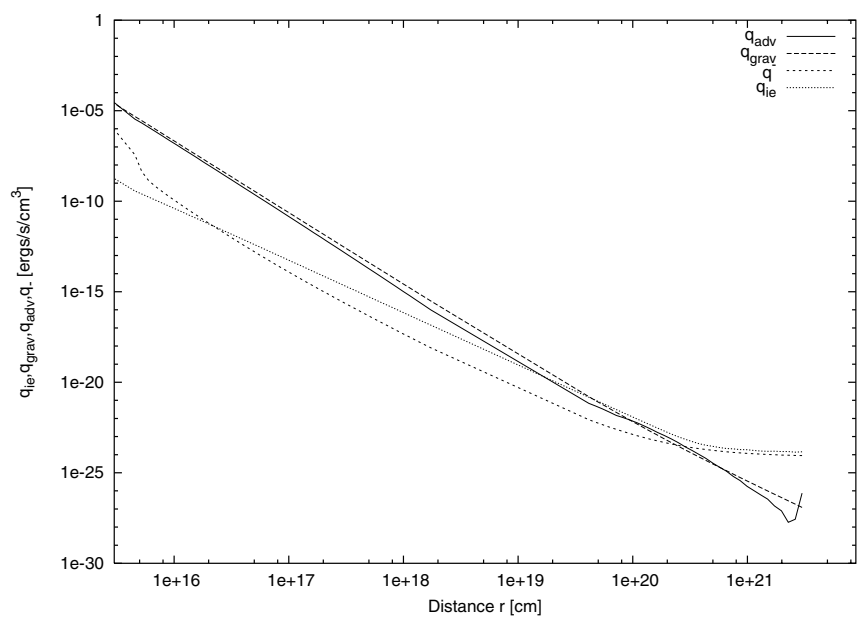

Fig. 4. Heating and cooling terms as a function of radius for parameter $\delta=0.1$ and $\beta=0.5$.

between electron temperature profiles is for $\delta=0.1$ and for $\delta=0.5$. The heating and cooling terms as a function of radius for $\delta=0.1$ are shown in Fig. 4. In this case the advection term do not dominate in the central parts of the flow. This time $Q_{\mathrm{adv}}$ is balanced by the compression heating term $\delta Q_{\text {grav }}$. In the outer part of the flow, $Q_{\text {ie }}$ and $Q^{-}$are balancing each other, the same as for $\delta=0.001$. The temperature converges to the same values at infinity. Radiation spectra for $\delta=0.001,0.01,0.1$, and 0.5 are presented in Fig. 6. This trend is similar to Fig. 5. For larger $\delta$, hotter electrons emit more synchrotron photons from the central parts of the flow. Bremsstrahlung emissivity in the spectrum is slightly affected by changing the $\delta$ parameter. The differences in $\log _{10}\left(L_{\mathrm{X}}\right)$, which for $\delta=0.001,0.01,0.1,0.5$ are $37.15,37.2,39.55,41.15$ respectively are mainly caused by the fact that the X-ray range is dominated by synchrotron photons scattered more than once.

\subsubsection{Results for different external medium conditions}

We also check whether the conditions of the external medium have an influence on the spectrum formation. The parameters 


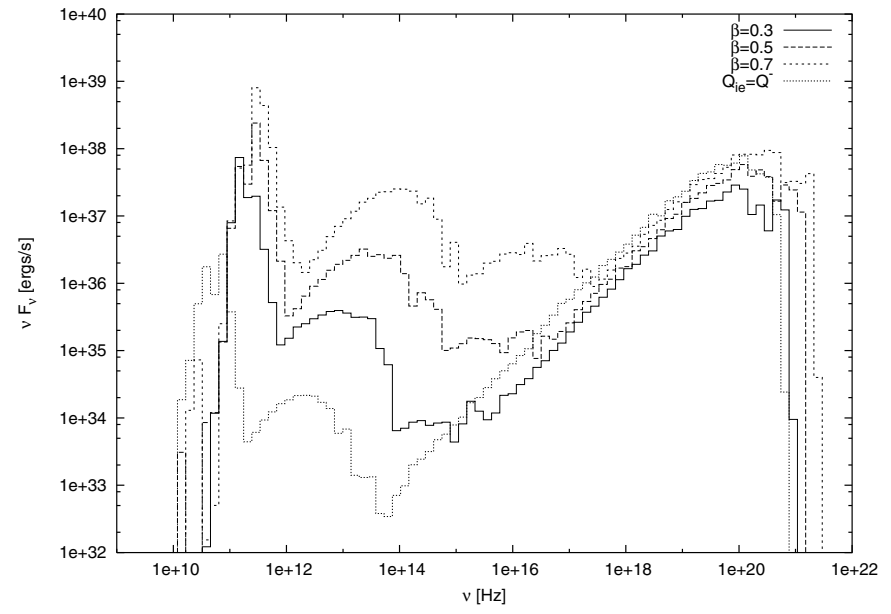

Fig. 5. Calculated spectra of $\mathrm{M} 87$ for model parameters $\beta=$ $0.3,0.5,0.7$

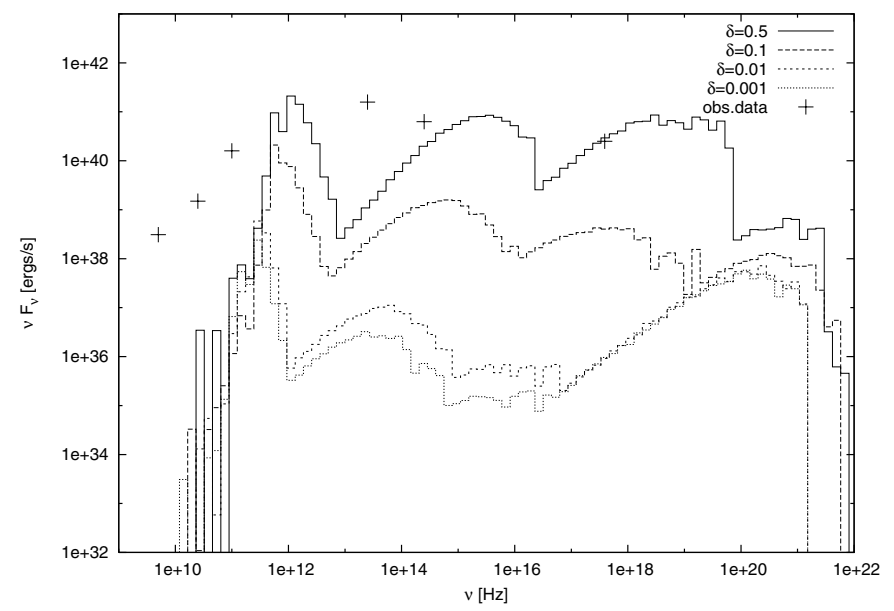

Fig. 6. Calculated spectra of M 87 for model parameters $\delta=$ $0.001,0.01,0.1,0.5$.

that we change are density of the external medium $\rho_{\infty}$ and external medium temperature $T_{\infty}$. By changing these parameters we change the structure of the flow and the mass accretion rate. We perform calculations for different $\rho_{\infty}$ and fix other parameter like $T_{\infty}, \beta$, and $\delta$. The density profiles for $\rho_{\infty}=0.1,1,10 \times$ $0.36 \times 10^{-24} \mathrm{~g} / \mathrm{cm}^{3}$ (M 87 external medium density value) are presented in Fig. 7, and the electron temperature profiles for these three cases are shown in Fig. 8. The electron temperatures remain almost the same in this case. The emitted spectra for these cases are presented in Fig: 11. The X-ray luminosities strongly depend on external medium density. By changing for the order of magnitude, we change the mass accretion rate's order of magnitude. As a result the whole of both spectra (both synchrotron and bremsstrahlung emissivities) rises for higher $\rho_{\infty} . \log _{10}\left(L_{X}\right)$ for $\rho_{\infty}=0.1,1,10 \times 0.36 \times 10^{-24} \mathrm{~g} / \mathrm{cm}^{3}$ are, 35.02, 37.15, 39.36 respectively.

In Fig. 9 we also show the effect on density profile of varying $T_{\infty}$ but keeping $\rho_{\infty}$ and spherical cloud boundary fixed. In general, density is higher at the central part of the flow for lower temperatures of the external medium. The electron temperatures convergence to similar values in the center.

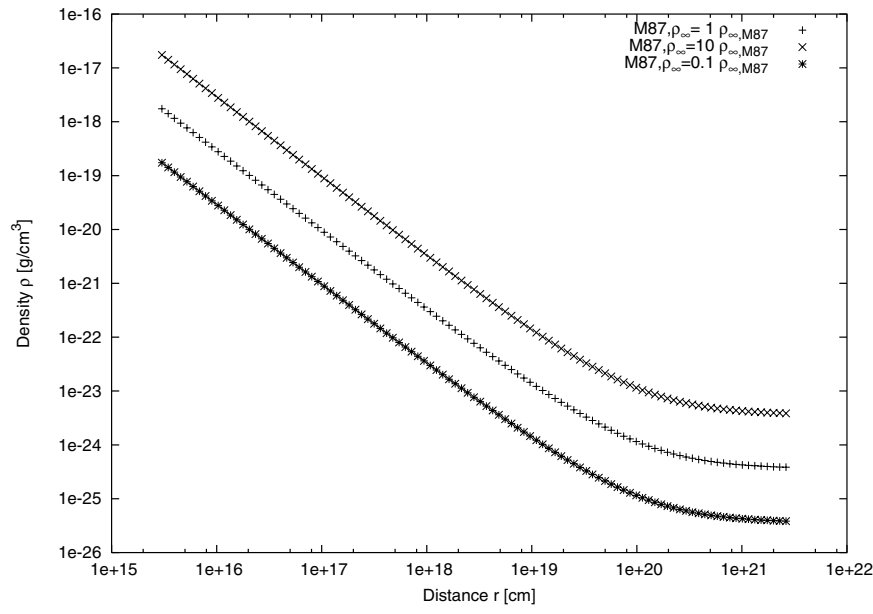

Fig. 7. Density profiles for different initial $\rho_{\infty}$, with calculations made for M 87.

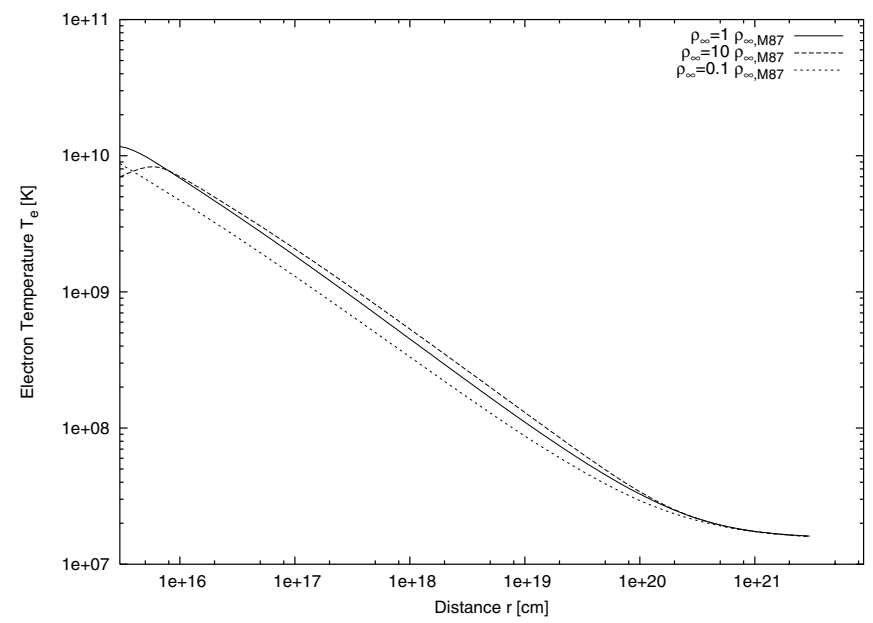

Fig. 8. Electron temperature profiles for different initial $\rho_{\infty}$, with calculations made for M 87.

In this case, radiation spectra are calculated assuming outer radius to be equal Bondi radius. The whole radiation spectrum decreases for higher $T_{\infty}$. The effect is greater for changing $T_{\infty}$, than for changing $\rho_{\infty}$. We also check whether the adopted outer boundary of the spherical cloud has a significant influence on the results. In Fig. 11 we also show spectra of M 87 for different boundary radius equal $10^{6} \times R_{\text {in }}$. This change affects only the bremsstrahlung part of the spectrum, which is created by the photons emitted from the outer shells of the spherical cloud. Then $\log _{10}\left(L_{X}\right)$ increases to $\log _{10}\left(L_{X}=38.24\right)$ by more than one order of magnitude. Synchrotron emissivity does not change, because it is created by the photons coming very close to the horizon. Small discrepancies are caused by the fact that we divide the flow into $N=100$ cells in radius, which gives a certain accuracy to the calculations. The larger the boundary radius we take, the higher the bremsstrahlung emissivity will be. 


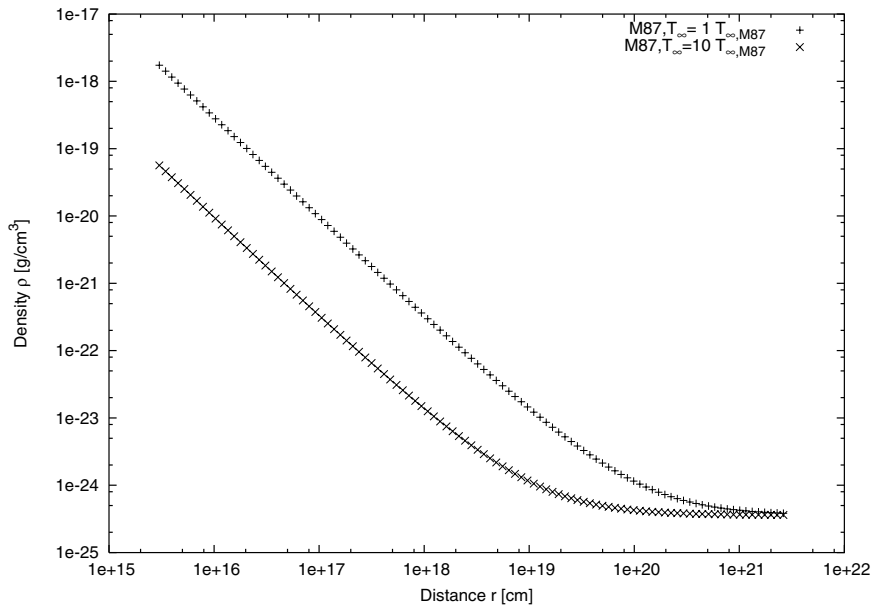

Fig. 9. Density profiles for different initial $k_{B} T_{\infty}$, with calculations made for M 87.

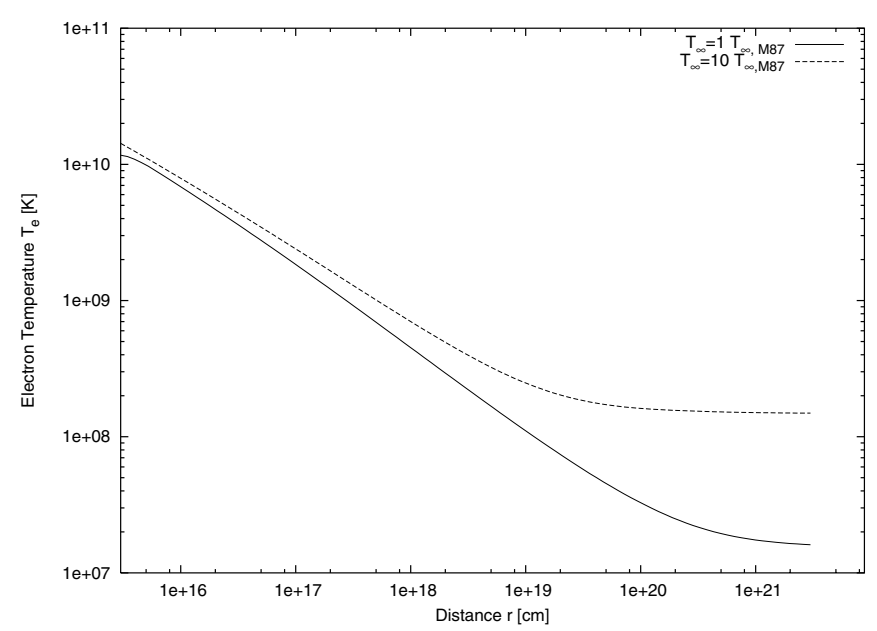

Fig. 10. Electron temperature profiles for different initial $k_{B} T_{\infty}$, with calculations made for M 87.

\subsection{Emerging spectrum and nuclear luminosities $L_{x}$ in LLAGN sample}

We calculate spectra for sample of 17 objects collected in P2005 assuming of the equipartition of the magnetic field $(\beta=0.5)$ and also assuming basic values $\delta=10^{-3}$ and $\delta=0.5$ for comparison, since the results strongly depend on this parameter. The assumed boundary radius of the scattering cloud is assumed to be Bondi radius $R_{\mathrm{Bondi}}$, which is proportional to external density medium $\rho_{\infty}$ and also depends on $T_{\infty}$. Thus $R_{\text {Bondi }}$ is different for each source. From obtained spectra we evaluated the expected X-ray luminosities $L_{\mathrm{X}}$ in the $0.3-10 \mathrm{keV}$ band. The results are presented in Table 1 . In most of the cases, modeled X-ray luminosities are much lower than observed if we assume that $\delta=10^{-3}$. In the cases of NGC 221 and NGC 5128, the difference is greater than 9 orders of magnitude. Sources that were under-predicted by 4-7 orders of magnitude are: NGC 1291, NGC 1316, NGC 1553, NGC 4261, NGC 4438, NGC 4594, NGC 4697, and IC 4296. For NGC 4472, M 87, and IC 1459 the difference between $L_{\mathrm{X}}$ and $L_{\mathrm{X} \text {,modeled ranges }}$ from 3-4 orders of magnitude, and $\mathrm{Sgr} \mathrm{A}^{*}$ is under-predicted about two orders of magnitude. Good estimation was achieved

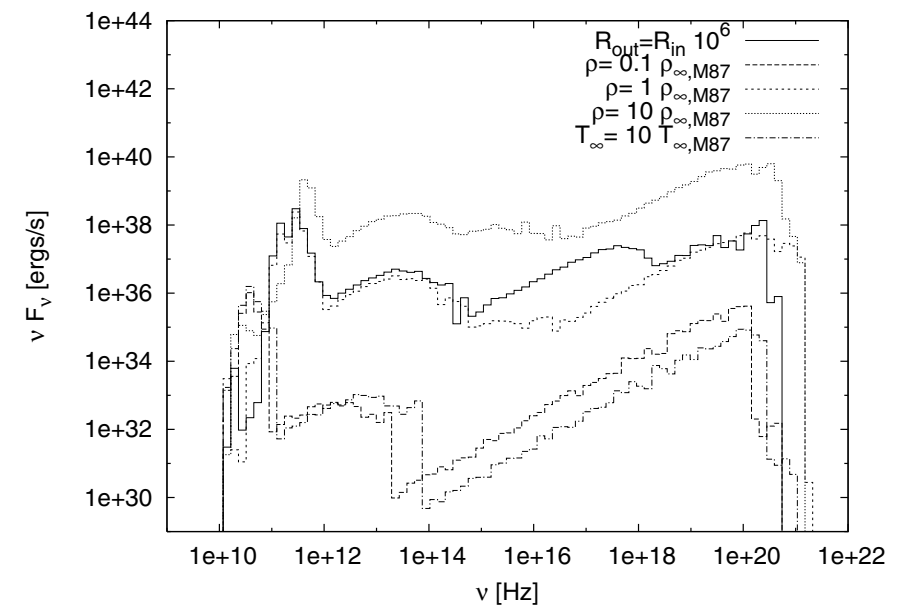

Fig. 11. Spectra for M 87 for different boundary conditions.

only for NGC 4649, for which observed X-ray luminosities are not larger than an order of magnitude from the modeled one. Three limitation values for NGC 821, NGC 1399, NGC 4636 are not exceeded.

In Table 2 we collect the characteristic properties of the sample of galaxies, to see whether the estimated values can be modified by including some additional effects. In the extreme case of NGC 5128(Cen A) where emitted X-ray luminosity differ by more than 9 orders of magnitude, our model is not appropriate. In this source calculated efficiency of accretion is $\eta=L_{\mathrm{X}, \text { observed }} / \dot{M}_{\text {Bondi }} c^{2}=0.32$. Because NGC 5128(Cen A) is an active galactic nucleus, given by $\mathrm{P} 2005$ for comparison, the result is reliable. The other source with a difference in modeled $L_{\mathrm{X}}$ and an observed one larger than 9 orders of magnitude is an elliptical dwarf galaxy with observed radio emission. In the case of sources for which $L_{X} / L_{X}$,modeled is between 4 and 7 orders of magnitude, observations may indicate of angular momentum inside the core: NGC 1291 is an early type spiral, NGC 1316 is disturbed early type elliptical, NGC 1553 has a spiral feature passing through a center, NGC 4261 core is surrounded by a disk of gas and dust, NGC 4438 has outflow bubbles that also may indicate angular momentum dominating the accretion flow, NGC 4594 is a spiral galaxy, and the last two sources NGC 4697 and IC 4296 are elliptical radio galaxies. Sources where $L_{X} / L_{X \text {,modeled }}$ is between 3 and 4 orders of magnitude, namely NGC 4472 and M 87, are elliptical radio galaxies with jets or radio lobes, while NGC 4649, which is modeled very well by our calculations, is an elliptical galaxy. Those sources for which we have only limitation values of X-ray emission are ellipticals. In NGC 4636 we see a spiral arm in the core.

For $\delta=0.5$ the results change dramatically. The efficiency of accretion grows 3 , even 5 , orders. One source, the Galactic center, differs more than 4 orders of magnitude from its observational values. For Galactic center the luminosity is over-predicted. The differences between modeled and measured $L_{\mathrm{X}}$ lie in a range 2-4 orders of magnitude for NGC 1553, NGC 4261, NGC 4649, and NGC 5128 (AGN). NGC 4649 is overpredicted more than 3 orders of magnitude. The modeled luminosity of NGC 5128 is too low in comparision with 
Table 1. Results of the simulation, assuming the equipartition of the magnetic field $(\beta=0.5), L_{\mathrm{X}}$ refer to a $0.3-10 \mathrm{keV}$ band, and it is given in logarithmic scale. ${ }^{*}$ For references see P2005; ${ }^{* *}$ our estimation of $L_{\mathrm{X}}$ (in logarithmic scale) emerging from Bondi accretion flow. $\eta^{* * *}$ calculated efficiency of spherical accretion defined as $\eta=L_{\mathrm{bol}} / \dot{M} c^{2} ;{ }^{x} \dot{m}=\dot{M} / \dot{M}_{\mathrm{Edd}}, \dot{M}_{\mathrm{Edd}}=L_{\mathrm{Edd}} / 0.1 c^{2}$.

\begin{tabular}{|c|c|c|c|c|c|c|c|c|c|}
\hline Source & $\begin{array}{c}M_{\mathrm{BH}} \\
\left(10^{8} M_{\odot}\right)\end{array}$ & $\begin{array}{l}k T_{\infty} \\
(\mathrm{keV})\end{array}$ & $\begin{array}{c}\rho_{\infty} \\
\left(10^{-24} \mathrm{~g} \mathrm{~cm}^{-3}\right)\end{array}$ & $\dot{m}^{x}$ & $\begin{array}{c}\text { Observed } \\
L_{\mathrm{X}, \text { nucl }} * \\
{[\mathrm{ergs} / \mathrm{s}]}\end{array}$ & $\begin{array}{c}\text { Cal. } L_{\mathrm{X}, \text { nucl }} * * \\
\delta=0.001 \\
{[\mathrm{ergs} / \mathrm{s}]}\end{array}$ & $\begin{array}{c}\eta^{* * * *} \\
\delta=0.001\end{array}$ & $\begin{array}{c}\text { cal. } L_{\mathrm{X}, \text { nucl }} * * \\
\delta=0.5 \\
{[\mathrm{ergs} / \mathrm{s}]}\end{array}$ & $\begin{array}{c}\eta^{* * * *} \\
\delta=0.5\end{array}$ \\
\hline NGC 221(M 32) & 0.025 & 0.37 & 0.13 & $3.1 \times 10^{-7}$ & 36.44 & 27 & $9.8 \times 10^{-9}$ & 33.89 & $2.4 \times 10^{-3}$ \\
\hline NGC 821 & 0.37 & 0.46 & 0.01 & $2.5 \times 10^{-7}$ & $\leq 38.66$ & 28.81 & $2.9 \times 10^{-9}$ & 33.41 & $3.1 \times 10^{-4}$ \\
\hline NGC 1291 & 1.1 & 0.34 & 0.56 & $6.7 \times 10^{-5}$ & 39.60 & 34.26 & $5.56 \times 10^{-7}$ & 38.78 & $1.9 \times 10^{-3}$ \\
\hline NGC 1316 & 3.9 & 0.62 & 0.44 & $7.6 \times 10^{-5}$ & 38.87 & 34.76 & $1.38 \times 10^{-7}$ & 38.3 & $1.6 \times 10^{-4}$ \\
\hline NGC 1399 & 12.0 & 0.8 & 0.47 & $1.6 \times 10^{-4}$ & $\leq 39.14$ & 35.94 & $1.9 \times 10^{-7}$ & 40.33 & $1.9 \times 10^{-3}$ \\
\hline NGC 1553 & 1.6 & 0.51 & 0.6 & $5.4 \times 10^{-6}$ & 40.01 & 34.15 & $1.47 \times 10^{-7}$ & 37.99 & 0.015 \\
\hline NGC 4261 & 5.4 & 0.6 & 0.17 & $4.2 \times 10^{-5}$ & 41.15 & 35.08 & $1.41 \times 10^{-7}$ & 39.11 & $3.9 \times 10^{-3}$ \\
\hline NGC 4438 & 0.5 & 0.58 & 0.99 & $2.4 \times 10^{-6}$ & 39.65 & 32.93 & $1.01 \times 10^{-7}$ & 38.39 & 0.02 \\
\hline NGC 4472 & 7.9 & 0.8 & 0.32 & $5.6 \times 10^{-5}$ & 38.69 & 35.10 & $1.74 \times 10^{-7}$ & 39.31 & $2.7 \times 10^{-3}$ \\
\hline NGC 4468(M 87) & 34.0 & 0.8 & 0.36 & $3.3 \times 10^{-4}$ & 40.88 & 37.13 & $2.92 \times 10^{-7}$ & 41.15 & $1.6 \times 10^{-3}$ \\
\hline NGC 4594 & 10.0 & 0.65 & 0.29 & $8.7 \times 10^{-5}$ & 40.34 & 35.6 & $1.5 \times 10^{-7}$ & 40.28 & $2.9 \times 10^{-3}$ \\
\hline NGC 4636 & 3.0 & 0.6 & 0.11 & $1.3 \times 10^{-5}$ & $\leq 38.41$ & 33.2 & $4.6 \times 10^{-8}$ & 38.44 & $3.0 \times 10^{-3}$ \\
\hline NGC 4649 & 20.0 & 0.86 & 1.05 & $8.1 \times 10^{-4}$ & 38.11 & 37.29 & $6.16 \times 10^{-7}$ & 41.37 & $4.9 \times 10^{-4}$ \\
\hline NGC 4697 & 1.7 & 0.33 & 0.05 & $9.7 \times 10^{-6}$ & 38.64 & 32.65 & $2.79 \times 10^{-8}$ & 37.62 & $2.1 \times 10^{-3}$ \\
\hline NGC 5128 & 2.4 & 0.5 & 0.08 & $1.0 \times 10^{-5}$ & 42.11 & 32.9 & $3.44 \times 10^{-8}$ & 38.21 & $3.0 \times 10^{-3}$ \\
\hline IC 1459 & 25.0 & 0.5 & 0.54 & $7.0 \times 10^{-4}$ & 41.18 & 37.7 & $7.95 \times 10^{-7}$ & 42.25 & $4.9 \times 10^{-5}$ \\
\hline IC 4296 & 11.0 & 0.56 & 1.0 & $6.7 \times 10^{-4}$ & 41.38 & 36.99 & $6.1 \times 10^{-7}$ & 41.16 & $7.0 \times 10^{-4}$ \\
\hline GC & 0.037 & 1.3 & 52.0 & $2.3 \times 10^{-5}$ & 33.38 & 31.21 & $1.1 \times 10^{-7}$ & 37.57 & $4.7 \times 10^{-4}$ \\
\hline
\end{tabular}

Table 2. Features of the galaxies in a calculated sample.

\begin{tabular}{|c|c|c|}
\hline Source & Features & Ref. \\
\hline NGC 221(M 32) & elliptical dwarf, radio emission & (1) \\
\hline NGC 821 & elliptical & (2) \\
\hline NGC 1291 & early type spiral & (3) \\
\hline NGC 1316 & disturbed early type elliptical, giant radio lobes, $\mathrm{S}$-shaped nuclear radio-jets & (4) \\
\hline NGC 1399 & elliptical & (5) \\
\hline NGC 1553 & spiral feature passing through the center, weak radio source & (6) \\
\hline NGC 4261 & elliptical radio galaxy, kiloparces scale jets, bright nuclear optical source surrounded by a disk of gas and dust & (7) \\
\hline NGC 4438 & spherical bulge, outflow bubbles in Northwest-South East direction from the nucleus & (8) \\
\hline NGC 4472 & giant elliptical galaxy, cavities (inner $2^{\prime}$ ) corresponding to the position of radio lobes & \\
\hline & two small extended sources within $10^{\prime \prime}$ of the nucleus of the galaxy, both the same luminosity & (9) \\
\hline NGC 4468(M 87) & elliptical radio galaxy, one-sided jet, large radio structure & $(10)$ \\
\hline NGC 4594 & spiral SAa type & $(11)$ \\
\hline NGC 4636 & elliptical, spiral arm in the core, cavity presence, hot gas violently disturbed & $(12)$ \\
\hline NGC 4649 & elliptical & (13) \\
\hline NGC 4697 & early type elliptical & (14) \\
\hline NGC 5128(Cen A) & AGN,giant elliptical radio galaxy, inner jet, radio-lobe & $(15)$ \\
\hline IC 1459 & $\begin{array}{l}\text { extremely radio-loud compared to normal radio-loud quasars, } \\
\text { giant elliptical galaxy, counter rotating core (merger possible) }\end{array}$ & (16) \\
\hline IC 4296 & giant elliptical radio galaxy, large-scale jets & (17) \\
\hline GC & shows flaring & (18) \\
\hline
\end{tabular}

(1) Ho et al. (2003), (2) Fabbiano et al. (2004); (3) Irwin et al. (2002); (4) Dong-Woo Kim et al. (2002); (5) Boute et al. (2002); (6) Blauton et al. (2001); (7) Zezas et al. (2005); (8) Machacek et al. 2004; (9) Biller et al. (2004); (10) Di Matteo et al. (2003); (11) Pellegrini et al. (2003a); (12) O'Sullivan et al. (2005); (13) Randall et al. (2004); (14) Sarazin et al. (2001); (15) Evans et al. (2004); (16) Fabbiano et al. (2003); (17) Pellegrini et al. (2003b); (18) Yuan et al. (2002), Senda et al. (2005)

observations. Two sources, NGC 1553, and NGC 4261 are still under-predicted by about 2 orders of magnitude. Other sources (NGC 1291, NGC 1316, NGC 4438, NGC 4472, M 87, NGC 4594, NGC 4697, IC 1459, IC 4296) differ from their observational counterparts about 1 order of magnitude, lots of them even less than 1 order of magnitude (see Table 1). The limitation value for NGC 821 is not exceeded, and it is still possibly 5 orders of magnitude under-predicted. In the case of NGC 1399, the modeled luminosity is an order of magnitude too high for the limitation value. For NGC 4636 it is also 
exceeded, but it is very close to its limit. The source that fit well to observations for $\delta=0.001$, NGC 4649, is now more than 3 orders of magnitude over-predicted.

\section{Discussion and conclusions}

In this paper we reconsider the sample of 17 LLAGNs (with measured temperature and density of the surrounding medium) and GC collected of P2005. Usually LLAGN are discussed in the context of the ADAF model (Quataert 1999; Di Matteo et al. 1999, 2003; Loewenstein et al. 2001). In general ADAF over-predicts measured luminosities, if one assumes that the mass accretion rate of the flow is equal to the Bondi mass accretion rate. As pointed out by Narayan (2002; also Quataert 2003), one should include the mass accretion rate reduced by the $\alpha$ factor (dimensionless viscosity parameter) in calculations, so that $\dot{M}_{\mathrm{ADAF}}=\alpha \dot{M}_{\mathrm{Bondi}}$, to make the model selfconsistent. Although the ADAF model, with proper assumption of $\dot{M}$, predicts X-ray luminosities in some cases well, it can over-predict the emission in radio band (Loewenstein 2001). Also for the sources with jet structures, it is hard to include additional emission in the radio band (Narayan 2002). The second point concerns estimation of the Bondi mass accretion rate $\dot{M}_{\text {Bondi }}$, which may be an order of magnitude smaller depending on the polytropic index $\gamma$ and mean particles mass $\mu$. For example for NGC 1399 the mass accretion rate assuming $\gamma=5 / 3$ and $\mu=0.5$ is $\dot{M}_{\text {Bondi }}=4.6 \times 10^{-3} M_{\odot} /$ yr, but if we assume different $\gamma=1.4$ (which is typical of the partially ionized ISM and usually assumed in ADAF models) and different $\mu=1.0$, we obtain an order of magnitude higher mass accretion rate $\dot{M}_{\text {Bondi }}=4.2 \times 10^{-2} M_{\odot} /$ yr. One should pay particular attention when choosing "constants" that determine accretion rate, because emerging spectrum depends strongly on this quantity. In our calculations we take $\gamma \approx 5 / 3$ and $\mu=0.5$.

Narayan (2002) also points out that LLAGN weak luminosities can be fitted to the data by assuming a two-temperature Bondi model. In this paper we apply a spherically symmetrical Bondi model of accretion to the sample of sources from P2005, and calculate the radiation spectra emerging from accreting plasma, including a full treatment of radiation transfer through the gas. Historically, the problem of spherical accretion onto a compact object has been present in many papers (for a review see Nobili et al. 1991). Although the model of steady spherical flow is only mathematical (not realized in nature because there is always some angular momentum present), it can be used as a rough approximation of an accretion with very small angular momentum, as we would expect to be present in elliptical galaxies. A similar model of spherical accretion was tested by Yim \& Park (1995) in the case of our Galactic center ${ }^{1}$. Melia (1994) considered semi-spherical flow (with very low angular momentum) but with the disc inside. The authors of both papers conclude that spectra can be reasonably explained with such assumptions.

Our calculations also show that spherical accretion can reconstruct observed X-ray luminosities in most cases of the

\footnotetext{
1 Detailed comparison to Yim \& Park (1995) is not possible because the paper is unavailable.
}

LLAGN sources presented in P2005, within an order of magnitude error (10 cases in 17 galaxies in a sample), for parameter $\delta=0.5$. The nuclear luminosities $L_{\mathrm{X}}$ are very sensitive to the changes in the heating parameter $\delta$. This parameter indicates how much accretion energy will be transfered to the electrons, and determines the electron temperature profile, while the $\beta$ parameter does not influence the electron temperature significantly. For most of the sources X-ray luminosities are strongly under-predicted when $\delta=0.001$. The obtained efficiency $\eta$ of spherical accretion in all cases for this value of $\delta$ is very small, on the order of $10^{-7}-10^{-9}$. If we assume $\delta=$ 0.5 , the electrons are heated to the same degree as ions. Half of the accretion energy is transferred to ions, the other half to the electrons. But the increase in X-ray luminosity is not caused by the growth of bremsstrahlung emissivity. When the temperature rises, the bremsstrahlung emission goes up like $T^{0.5}$ and is more sensitive to the changes in density (like $\rho^{2}$ ) than to the electron temperature, so the effect is not very strong for the emissivity of bremsstrahlung radiation. The reason for this increase in $L_{X}$ is that the synchrotron emissivity grows, and the X-ray part of the spectrum is dominated by the Compton-scattered synchrotron photons. The efficiency even grows 5 orders of magnitude for $\delta=0.5$. Our calculations show that the assumption that electrons are heated directly by accretion to the same degree as ions is more appropriate than assuming $\delta$ to be 0.001 because of mass ratio $\left(m_{\mathrm{e}} / m_{\mathrm{p}}\right)$ (e.g. Esin et al. 1997; Manmoto 2000) This agrees with the results of Bisnovatyi-Kogan \& Lovelace (1997), who argue that in the presence of a magnetic field and plasma instabilities, gravitational energy is transfered predominantly to electrons. Also in Yuan et al. (2003b) the assumption of $\delta=0.55$ allows accommodation of the spectrum of Sgr A* with the RIAF model.

Bremsstrahlung emissivity depends strongly on the external medium conditions $\left(T_{\infty}\right.$ and $\left.\rho_{\infty}\right)$ that control the mass accretion rate. The values of these quantities are estimated by observations, so they cannot be changed significantly in this approach. In the case of over-predicted sources, $L_{X}$ can be reduced and accommodated to observations with the assumption of an accretion with outflows, which was considered by e.g. Quataert \& Narayan (1999). For one of the sources the Galactic center, nuclear luminosity $L_{\mathrm{X}}$ is strongly over-predicted by our model when $\delta=0.5$. External medium measurements indicate (assuming Bondi flow) that the mass accretion rate for this source is $\sim 10^{-6} M_{\odot} /$ yr. On the other hand, we also have mass accretion rate limitations estimated by measurements of polarization of radiation near the black hole in Sgr A*. From Faraday rotation we obtain a limit for $\dot{M} \sim 10^{-7} M_{\odot} /$ yr (Atiken et al. 2000). This fact also eliminates a simple ADAF model for this source (Bower et al. 2005). Differences between $\dot{M}$ near the capture radius and $\dot{M}$ near the horizon indicate that most of the accreting material does not reach a black hole. For Sgr A* the possible explanation could be the accretion flow disturbed by the outflow. For $\mathrm{Sgr} \mathrm{A}^{*}$ the radio part of a spectrum is too high in our model for $\delta=0.5$, and it is also higher than the NIR limitations for the mass accretion rate $\dot{M} \sim 10^{-6} M_{\odot} / \mathrm{yr}$. The outflow could both be a possible explanation of the spectrum and also reduce the mass accretion rate. For $\delta=0.001$ the emission is very low, the peak of the synchrotron radiation 
attain in $v F_{v}=10^{34}$ [ergs/s]. The ADAF model of Narayan et al. (1998) gives a the denser accretion flow than the Bondi flow, since the radial velocity is smaller. Thus our results for $\delta=0.001$ are much lower the observational points. In Yuan et al. (2003b), the emission also is accommodated to Xray emission in the assumption of a higher $\delta=0.55$ value, but only for $\dot{M}=10^{-8} M_{\odot} /$ yr. The reason our model for Sgr A* gives such poor constraints for $\delta=0.5$, in comparison with Yuan et al. (2003a), is that we assume a Bondi accretion rate that is two orders of magnitude higher than $\dot{M}=10^{-8} M_{\odot} / \mathrm{yr}$. Our model can reproduce the Sgr A* spectrum better if we allow for the specific adjustment of the parameters like $\dot{M}$ and $\delta$, since the synchrotron emissivity is very sensitive to the temperature changes (controlled mainly by these two parameters). Our model can also explain the soft spectral slope seen in the data (Baganoff et al. 2003), since the Comptonized synchrotron component can extend to the X-ray band. However, the model never fits the radio frequencies below $\sim 10^{11} \mathrm{~Hz}$. This part of the spectrum can only be explained by models that include nonthermal populations of electrons, such as the RIAF-jet model of Yuan et al. (2003b). The presence of a jet-like outflow also in other sources is supported by observations (see Table 2). Also the negligence of the spherically symmetric outflow is a serious weakness of our model since there is now observational evidence that the accretion rate for Sgr A* is not constant with radius (e.g. Bower et al. 2003).

The effect of the angular momentum of the accretion flow also cannot be neglected, the low angular momentum was considered by Proga et al. (2003), but without any estimations of emerging spectra. The Bondi spherical accretion model is a mathematical model. In reality there is always angular momentum. We also know that standing shocks are the part of low angular momentum flows (Das 2002; Das et al. 2003). Also turbulent flow may create shocks. In our model we assume the thermal distribution of electrons. This is a weakness of the model, since the shock produces some fraction of non-thermal electrons. Thanks to studies of Sgr A* (Mahadevan 1999; Ozel et al. 2000; Yuan et al. 2003b) with hybrid (thermal + nonthermal power-law tail) population of electrons, we know that the observed low-frequency radio spectrum of Sgr $\mathrm{A}^{*}$ can be explained if a small fraction of electrons is non-thermal. Close to a black hole, turbulence and magnetic reconnection can accelerate electrons. Synchrotron emission from these electrons and Compton scattering on them can be a reason for flaring in broadband spectrum. In all calculations of spectra for M 87, the low-frequency radio emission is not reconstructed, which may be a reason that non-thermal electrons should be included in calculations; moreover, scattered nonthermal photons can affect the X-ray band of the spectrum.

Our model is also not consistent with recent results of modeling spherical accretion with magnetic fields. Because we include synchrotron emission in calculating the radiation spectrum, we assume that there is some random magnetic field in plasma surrounding a black hole. Spherical flows including magnetic field are unstable (e.g. Igumenshchev et al. 2002), thus we are able to obtain only some mean luminosity of spherical accretion. Our model is not able to reconstruct any detailed features of time-dependent spectra like e.g. flares. Another disadvantage of our model is that the equations of spherically symmetrical flow are calculated in the Newtonian regime. Including relativistic effects may be important very close to a horizon of a black hole, where in our calculations most of the synchrotron radiation comes from.

Acknowledgements. We would like to thank Bozena Czerny, Aneta Siemiginowska, Piotr Zycki, and Agata Rozanska for helpful comments and useful discussions. Part of this work was supported by grant PBZ-KBN-054/P03/2001 and 1P03D00829 of the Polish State Committee for Scientific Research.

\section{References}

Atiken, D. K., Greaves, J., Chrysostomou, A., et al. 2000, ApJ, 534, L173

Baganoff, F. K., Maeda, Y., Morris, M., et al. 2003, ApJ, 591, 891

Biller, B. A., Jones, C., Forman, W. R., Kraft, R., \& Ensslin, T. 2004, ApJ, 613, 238

Bisnovatyi-Kogan, G. S., \& Lovalace, R. V. E. 1997, ApJ, 486, L43

Blauton, E. L., Sarazin, C. L., \& Irwin, J. A. 2001, ApJ, 552, 106

Bondi, H. 1952, MNRAS, 112, 195

Bower, G. C., Wright, M. C. H., Falcke, H., \& Backer, D. C. 2003, ApJ, 588, 331

Bower, G. C., Falcke, H., Wright, M. C. H., \& Backer, D. C. 2005, ApJ, 618, L29

Buote, D. A. 2002, ApJ, 574, L135

Camenzind, M. 2005, MnSAI, 76, 98

Das, T. K. 2002, ApJ, 577, 880

Das, T. K., Pendharkar, J. K., \& Mitra, S. 2003, ApJ, 592, 1078

Di Maatteo, T., Fabian, A. C., Rees, M. J., Carilli, C. L., \& Ivison, R. J. 1999, MNRAS, 305, 492

Di Matteo, T., Allen, S. W., Fabian, A. C., Wilson, S., \& Young, A. J. 2003, ApJ, 582, 133

Dong-Woo, Kim, \& Fabbiano, G. 2003, ApJ, 586, 826

Esin, A. A., Narayan, R., Ostricker, E., \& Yi, I. 1996, ApJ, 465, 312

Esin, A. A., McClintock, J. E., \& Narayan, R. 1997, ApJ, 489, 865

Evans, I. N., \& Koratkar, A. P. 2004, ApJ, 617, 209

Fabbiano, G., Elvis, M., Markoff, S., et al. 2003, ApJ, 588, 175

Fabbiano, G., Baldi, A., Pellegrini, S., et al. 2004, ApJ, 616, 730

Genzel, R., Schoedel, R., Ott, T., et al. 2003, Nature, 425, 934

Gorecki, A., \& Wilczewski, W. 1984, AcA, 34, 141

Ho, L. C. 2003, ASPC, 290, 379

Ho, L. C., Terashima, Y., Ulvestad, J. S. 2003, ApJ, 589, 783

Igumenshchev, I. V. 2002, ApJ, 566, 137

Irwin, J. A., Sarazin, C. L., \& Bregman, J. W. 2002, ApJ, 570, 152

Kurpiewski, A., \& Jaroszynski, M. 2001, A\&A, 346, 713

Loewenstein, M., Mushotzky, R. F., Lorella, A., Arnaud, K. A., \& Quataert, E. 2001, ApJ, 555, 21L

Machacek, M. E., Jones, C., \& Forman, W. R. 2004, ApJ, 610, 183

Mahadevan, R. 1999, MNRAS, 304, 501

Mahadevan, R., Narayan, R., \& Yi, I. 1996, ApJ, 465, 327

Manmoto, T. 2000, ApJ, 534, 734

Melia, F. 1994, ApJ, 426, 577

Narayan, R. 2002, Lighthouses of the Universe: The Most Luminous Celestial Objects and Their Use for Cosmology Proceedings of the MPA/ESO/, 405

Narayan, R., Yi, I., \& Mahadevan, R. 1995, Nature, 374, 623

Narayan, R., Mahadevan, R., Grindlay, J. E., Popham, R. G., \& Gammie, C. 1998, ApJ, 492, 554

Nayakshin, S. 2004, MNRAS, 352, 1028

Nobili, L., Turolla, R., \& Zampieri, L. 1991, ApJ, 383, 250 
O’Sullivan, E., Vrtilek, J. M., \& Kempner, J. C. 2005, ApJ, 624, L77 Ozel, F., Psaltis, D., \& Narayan, R. 2000, ApJ, 541, 234

Pacholczyk, A. G. 1970, Radio Astrophysics (San Francisco: Freeman)

Pellegrini, S. 2005, ApJ, 624, 155 (P2005)

Pellegrini, S., Boldi, A., Fabbiano, G., \& Kim, D.-W. 2003a, ApJ, 597, 175

Pellegrini, S., Venturi, T., Comatsu, A., et al. 2003b, ApJ, 585, 677

Pozdnyakov, L. A., Sobol, I. M., \& Syunyaev, R. A. 1983, ASPR, 2, 189

Proga, D., \& Begelman, M. C. 2003, ApJ, 592, 767

Ptak, A., Yaqoob, T., Mushotzky, R., Serlemitsos, P., \& Griffiths, R. 1998, ApJ, 501, L37

Quataert, E. 2003, Astron. Nachr./AN 324, No. S1

Quataert, E., \& Narayan, R. 1999, ApJ, 520, 298
Randall, S. W., Sarazin, C. L., \& Irwin, J. A. 2004, ApJ, 600, 729

Rozanska, A., \& Czerny, B. 2000, A\&A, 360, 1170

Sarazin, C. L., Irwin, J. A., \& Bregman, J. N. 2001, ApJ, 556, 533

Senda, A., Takagi, S., Koyama, K., Bamba, K., \& Murakami, H. 2005, X-ray and radio connections, ed. L. O. Sjouwerman, \& K. K. Dyer, Published electronically by NRAO,

http://www. aoc.nrao.edu/events/xraydio

Yim, Soo Youn, \& Park, Meyong-Gu 1995, PKAS, 10, 79

Yuan, F., Markoff, S., \& Falcke, H. 2002, A\&A, 383, 854

Yuan, F., Markoff, S., Falcke, H., \& Bierman, P. L. 2003a, New AR, 47,705

Yuan, F., Quataert, E., \& Narayan, R. 2003b, ApJ, 598, 301

Zezas, A., Birkinshaw, M., Worrall, D. M., Peters, A., \& Fabbiano, G. 2005, ApJ, 627, 711 